Richard

P. MacDermott

Gary R. Lichtenstein Ryo Izutani

Toshio Muraki

\title{
Anomalies \\ du système immunitaire de la muqueuse au cours des maladies inflammatoires de l'intestin
}

L'immunopathologie des maladies inflammatoires cryptogénétiques de l'intestin comporte une cascade d'étapes immunologiques, initiée par la captation et la préparation d'antigènes et suivie du développement du processus inflammatoire. La nature des antigènes reste inconnue, mais il est possible que des produits d'origine bactérienne jouent un rôle dans l'initiation et/ou la pérennisation d'un processus immunologique non contrôlé responsable des lésions intestinales chez des sujets génétiquement prédisposés. Macrophages et lymphocytes $T$ sont anormalement activés et les immunoglobulines, surtout les IgG, sont produites en excès localement, sous l'effet des cytokines. La cascade inflammatoire implique les macrophages, les polynucléaires et de nombreux médiateurs dont les leucotriènes, le facteur d'activation plaquettaire et les radicaux libres. Les cellules cibles intestinales peuvent être détruites, soit de façon non spécifique par les médiateurs de l'inflammation, soit par un processus auto-immun à médiation cellulaire directe et dépendante des anticorps.

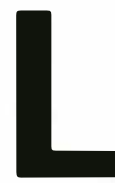

'immunopathologie des maladies inflammatoires cryptogénétiques de l'intestin correspond à une cascade d'étapes immunologiques avec pour point de départ les réactions initiales du traitement des antigènes [1-3]. La nature précise des antigènes qui déclenchent les maladies inflammatoires cryptogénétiques de l'intestin (MICI) demeure inconnue. Cependant, on a porté récemment une grande attention aux produits des parois cellulaires bactérien- nes, tels que les protéoglycanes, la formyl-méthionyl-leucyl phénylalanine (fMLP) et les lipopolysaccharides (figure 1). Ces produits induisent, de façon non spécifique, une intense activation des macrophages, des granulocytes et des lymphocytes. Ainsi, les bactéries, par les effets des produits de leurs parois cellulaires, ou par leurs toxines, jouent-elles un rôle important dans l'initiation et la pérennisation des MICI (figure 1). L'hypothèse de travail actuelle est que des produits des membranes cel- vard, Philadelphia, PA 191046144 , États-Unis. 


\section{RÉFÉRENCES}

1. Macl)ermott RP. Cell-mediated immu nity in gastrointestinal discasc. Hum Patho $1986 ; 17: 219-33$

2. Macl)ermott RP, Stenson WF. Alterations in the immune system in ulcerative colitis ans Crohn's discase. Adv Immunol $1988 ; 42$ : 285-328.

3. Macl)ermott RP, Stenson WF. The role of the immune system in inflammatory bowel discasc. Immunol Allerg Clin North Am $1988 ; 8: 521-42$.

4. Brandtzacg P, Nilssen IDE, Rognum T(), Thrane PS. Ontogeny of the mucosal immunc system and IgA deficiency. Gastro enterol Clin North Am 1991; 20 : 397-441.

5. Trier JS. Structure and function of intes tinal M cells. Gastroenterol Clin North $A m$ $1991 ; 20: 531-48$.

6. Wolf JI, Rubin IH, Finberg R, et al. Intestinal $M$ cells : a pathway for entry of reovirus into the host. Science $1981 ; 212$ 471-2.

7. Bienenstock J, MacDermott M, Befus D) et al. A common mucosal immunologic system involving the bronchus, breast, and bowel. Adv Exp Med Biol 1978; 107 : 53-9. 8. Cerf-Bensussan N, Guy-Grand D. Intestinal intracpithelial lymphocytes. Gastroenterol Clin North Am 1991; 20 : 549-76.

9. Ernst PB, Befus AI), Bienenstock J. I eu cocytes in the intestinal epithelium : an unusual immunological compartment. Immunol Today $1985 ; 6$ : 50-5.

10. Bonneville $\mathrm{M}$, Janeway CA, I to $\mathrm{K}$, et al. Intestinal intracpithelial lymphocytes are a distinct set of gamma-delta T cells. Nature 1988 ; 336 : 479-81

11. Goodman 'T', Lefrancois L. Expression of the gamma-delta $\mathrm{T}$-cell receptor on intestinal CI 8 + intracpithelial lymphocytes. Nature 1988, 333 : 855-8.

12. Brandtzacg $P$, Bosnes B, Halstensen TS, et al. T lymphocytes in human gut cpithelium preferentially express the alpha/beta antigen receptor and are often CI)45/UCHL1-positive. Scand I Immuno $1989 ; 30$ : 123-8

13. Spenser I, Isaacson PG, Diss TC, MacDonald TT. Expression of disulfide-linked and non-disulfide-linked forms of the T-cel receptor $\gamma / \delta$ heterodimer in human intracpithelial lymphocytes. Eur J Immunol 1989 19 : 1335-8.

14. Fukushima K, Masuda T, Ohtani H, et al. Immunohistochemical characterization, distribution and ultrastructure of lymphocytes bearing $\mathrm{T}$ cell receptor $\gamma \delta$ in inflammatory bowel discasc. Gastroenterology 1991 ; 101: 670-8

15. Mestecky J. The common mucosal immune system and current strategies for induction of immune responses in external sccretions. J Clin Immunol 1987 ; 7 : 265-76. 16. Hanson LA, Ahlstedt S, Anderson B, et al. The biologic propertics of secretory IgA. J Reticuloendothel Soc 1980 ; 23 (suppl) $1-9$.

17. Jalkanen S, Reichert RA, Gallatin WM, et al. Homing receptors and the con trol of lymphocyte migration. Immunol Rev 1986 ; $91: 39-60$. lulaires bactériennes, capables de déclencher une réponse immune inflammatoire, activent, chez des sujets génétiquement prédisposés, une série d'événements immunitaires échappant à une répression correcte [1-3]. Ces facteurs déclencheraient l'activation de macrophages, conduisant à la sécrétion de grandes quantités d'interleukine 1 (IL1), d'IL6, d'IL8 et de tumor necrosis factor $\alpha$ (TNF- $\alpha)$ (figure 1). Les réactions immunologiques à médiation cellulaire qui en découlent impliquent le système immunitaire de la muqueuse, aboutissant à la destruction intestinale chronique caractéristique des MICI [1-3].

Le tissu lymphoïde associé aux muqueuses digestives (gut associated lymphoid tissue, GALT) constitue le plus important tissu immunitaire du corps [4]. Des cellules épithéliales spécialisées, appelées cellules $\mathbf{M}$, recouvrent les plaques de Peyer et les follicules lymphoïdes. Elles assurent le transport spécifique, jusqu'au tissu lymphoïde (GALT) sous-jacent, des virus et des antigènes de grande taille qui vont interagir avec les lymphocytes et les macrophages [5, 6]. Après la présentation des antigènes aux lymphocytes immatures de certaines zones des plaques de Peyer, ceux-ci migrent hors de l'intestin, mûrissent $\mathrm{au}$ sein des systèmes lymphatique et sanguin ("voyage de maturation"), puis retournent dans la lamina propria [7]. L'intestin possède aussi une sous-classe unique de cellules, les lymphocytes intra-épithéliaux [8, 9]. Ce sont des cellules $\mathrm{T}$, à phénotype de cellule cytotoxique suppressive (CD8 + essentiellement), qui présentent également un type unique de récepteur de cellule $\mathrm{T}(\gamma / \delta)[10,11]$. Il est intéressant de noter que le nombre de lymphocytes intraépithéliaux [8, 9] et de cellules $\mathrm{T} \gamma / \delta[10,11]$ est augmenté de façon importante chez les patients ayant une entéropathie au gluten [12, 13], mais non chez ceux atteints de MICI [14]. Certains agents infectieux, tels les réovirus [6], adhèrent spécifiquement aux cellules M [5], mais n'adhèrent pas aux autres cellules de l'épithélium; elles peuvent donc être transportées à travers l'épithélium jusqu'aux follicules lymphoïdes et aux plaques de Peyer sous- jacentes, af in de permettre l'apprêtement de l'antigène et sa présentation aux macrophages et aux lymphocytes. Ainsi, contrairement à l'idée répandue que l'intestin présente une barrière très stricte vis-à-vis des antigènes, les cellules $\mathrm{M}$ font un échantillonnage des très nombreux agents microbiens présents dans la lumière intestinale, afin d'induire d'efficaces réactions de défense chez l'hôte. Les cellules $\mathrm{M}$ peuvent cependant servir aussi de porte d'entrée aux virus et bactéries pathogènes.

Les lymphocytes des plaques de Peyer sont immatures et, après une première présentation et sensibilisation de l'antigène, quittent l'intestin et migrent à travers les ganglions lymphatiques, les lymphatiques efférents, le canal thoracique, s'engageant dans un cycle de transformation en lymphoblastes mûrs [7, 15]. Ils circulent ensuite dans le sang périphérique et retournent dans la muqueuse gastro-intestinale ou dans d'autres sites sécrétoires [7, 15]. L'un des meilleurs exemples du processus de homing est celui des mères qui allaitent et auxquelles on fait ingérer des bactéries non pathogènes : on retrouve dans leur lait des anticorps spécifiques qui ont un effet protecteur vis-à-vis des bactéries administrées par voie orale [16]. L'immunisation passive contre les organismes entériques est ainsi transférée de la mère à l'enfant allaité $[7,15,16]$. Les mécanismes de homing des lymphoblastes [17-20] impliquent que les endothéliums veinulaires présentent à leur surface des antigènes spécifiques, appelés adressines [19], pour interagir avec les récepteurs de homing spécifiques d'organes des lymphoblastes mûrs. Les interactions à la surface des cellules entre lymphoblastes et endothélium veinulaire déterminent les sites où les lymphoblastes vont migrer. Des récepteurs spécifiques au niveau des plaques de Peyer ou des cellules endothéliales des follicules lymphoïdes intestinaux orientent les lymphoblastes pour qu'ils retournent vers des sites muqueux intestinaux, et non vers des sites ganglionnaires périphériques $[17,20]$. Les cellules mononucléées normales de la lamina propria, chez l'homme, se lient peu aux endothéliums veinulaires des ganglions périphériques, tandis qu'elles se lient 


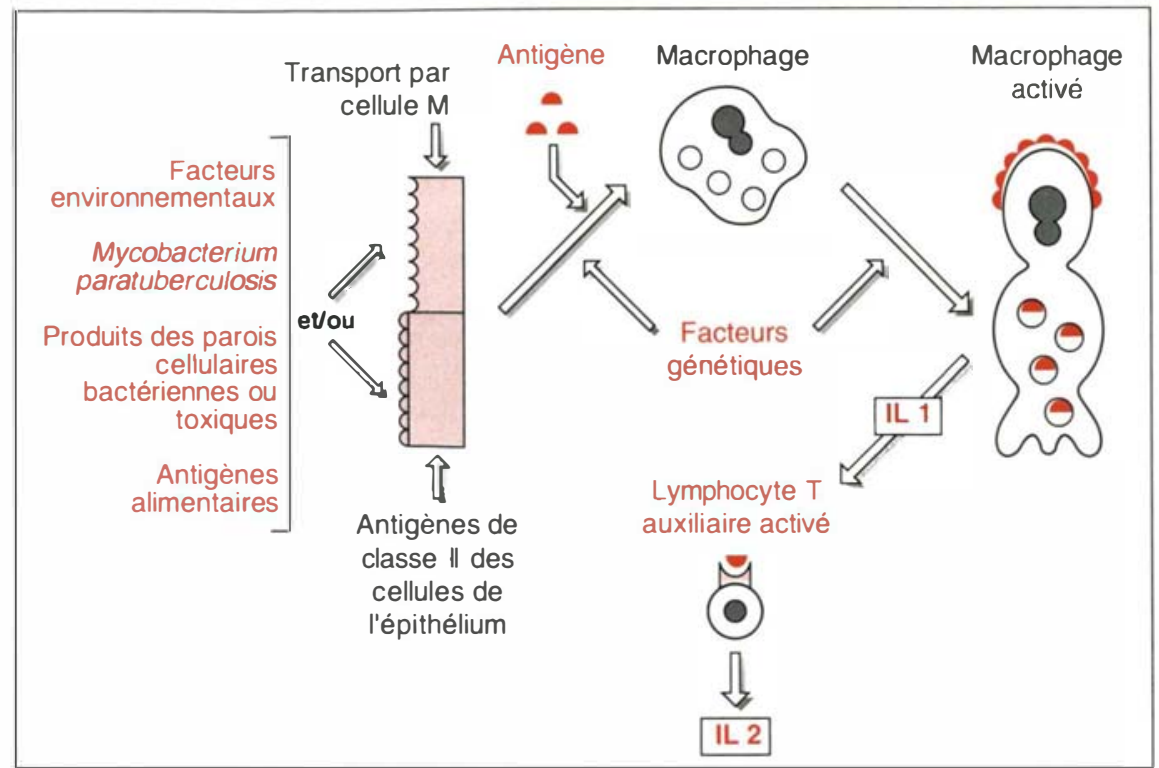

Figure 1. Facteurs déclenchants des maladies inflammatoires cryptogénétiques de l'intestin. Éléments environnementaux, mycobactéries, agents infectieux, produits de parois bactériennes, toxines, ou antigènes alimentaires peuvent être transformés par les cellules M. La présentation de l'antigène, associée aux antigènes de classe II sur les cellules épithéliales intestinales peut entraîner une réponse immunitaire intestinale chronique chez les malades souffrant de maladie intestinale inflammatoire chronique. Chez les sujets prédisposés génétiquement, les macrophages peuvent alors activer la réponse immunitaire en produisant des cytokines, telle I'IL 1. L'activation ultérieure des lymphocytes $T$ auxiliaires, associée à une production accrue d'IL2, peut alors déclencher des réactions à médiation cellulaire. Bien qu'on ne connaisse pas précisément les agents infectieux ou les antigènes luminaux responsables de la maladie inflammatoire chronique, le déclenchement d'une réponse immunitaire muqueuse est peut-être un mécanisme de défense approprié contre un agent pathogène, mais incapable de le faire disparaître. Alternativement, serait malade celui qui déclencherait une réponse inflammatoire d'une vigueur inappropriée contre une bactérie banale ou des antigènes alimentaires. (Modifié d'après [47].)

très bien aux endothéliums veinulaires de la muqueuse intestinale [20]. De grandes cellules lymphoblastiques de l'intestin humain normal, qui portent de façon prédominante une IgA (environ $60 \%$ sont $\operatorname{Ig} \mathrm{A}+$ ), et des cellules $\mathrm{T}$ avec des récepteurs pour le fragment $\mathrm{Fc}$ des $\mathrm{IgA}$, forment un sous-groupe cellulaire de la lamina propria et se lient avec avidité aux endothéliums veinulaires de la muqueuse [20]. Il semble donc y avoir, chez l'homme comme chez l'animal, des cellules dans la lamina propria qui présentent à leur surface des récepteurs de homing pour guider les cellules du système immunitaire muqueux vers les sites intestinaux. A mesure que s'enrichissent nos connaissances sur les relations entre système immuni$\mathrm{m} / \mathrm{s} n^{\circ} 8.9$ vol. 9, aoủt-septembre 93 taire muqueux et MICI, nous devrions mieux comprendre les mécanismes de homing des lymphoblastes impliqués dans la migration spécifique des lymphoblastes vers les sites de l'inflammation au cours de la rectocolite hémorragique et de la maladie de Crohn [19].

Plusieurs voies thérapeutiques visant à stopper les facteurs déclenchants de l'inflammation gastro-intestinale pourraient être développées. Les produits des parois cellulaires bactériennes (peptidoglycanes, fMLP, lipopolysaccharides) sont les premiers à stimuler la réponse intestinale immunitaire et inflammatoire. Il serait donc important d'explorer les possibilités d'interférer avec les effets proinflammatoires de ces produits bacté- riens généralement présents dans la lumière intestinale. En interférant avec les récepteurs des cellules $\mathbf{M}$, on pourrait aussi bloquer le système de transport de molécules qui permet le traitement direct des antigènes par les cellules du sytème immunitaire muqueux. Mais, il faudra en savoir beaucoup plus sur les mécanismes physiologiques de défense de l'organisme et sur le système immunitaire de la muqueuse pour pouvoir interférer avec les voies d'inhibition du trafic cellulaire, de l'activation des lymphocytes et du homing des lymphoblastes. Enfin, la régulation de la croissance cellulaire de l'épithélium et de son renouvellement représente une nouvelle voie thérapeutique intéressante axée sur la cicatrisation dans les maladies inflammatoires cryptogénétiques de l'intestin (MICI).

Les mécanismes de la destruction cellulaire comprennent l'atteinte non spécifique de cellules cibles, "spectatrices innocentes", par des médiatcurs de l'inflammation, ainsi qu'une atteinte auto-immune de l'intestin à médiation cellulaire directe et dépendante des anticorps : les facteurs responsables des lésions sont donc relayés à la fois par des cellules à mémoire et par des facteurs non spécifiques de l'inflammation [1-3]. La mémoire immunologique cellulaire et les facteurs spécifiques au cours des MICI déterminent une cascade d'événements soigncusement régléc (figure 2). La production de grandes quantités d'IL1, d'IL6 et de TNF$\alpha$ [21-23] par les macrophages et/ou d'autres types cellulaires, permet l'activation des cellules $T$ qui entraîne la stimulation clonale de nombreuses sous-classes de cellules T. La production d'IL2 est également augmentée dans les lésions des MICI mais, curieusement, les études réalisées in vitro ont montré une diminution du nombre des cellules sécrétant l'IL2 au sein des lymphocytes infiltrant la muqueuse [24]. La production de cytokines stimule le développement des cellules $T$ auxiliaires, et la synthèse et la sécrétion d'anticorps qui en résultent stimulent encore la fonction immunitaire. L'activation 


\section{RÉFÉRENCES}

18. Jalkanen S, Strecter P, Lakcy E, et al. L.ymphocyte and endothelial cell recognition clements that control lymphocyte traffic to mucosa-associated lymphatic tissues. Monogr Allergy 1988 ; 24 : 144-9.

19. Strecter PR, Berg EL, Rousc BTN, et al. A tissuc specific endothelial cell moleculc involved in lymphocyte homing. Nature 1988; 331: 41-6.

20. Salmi M, Jalkanen S. Regulation of lymphocyte traffic to mucosa-associated lymphatic tissues. Gastroenterol Clin North $\mathrm{Am}$ $1991 ; 20$ : 495-510.

21. Brynskov J, Tuede N, Andersen CB Vilien M. Increased concentration of interleukin $1 \beta$, interleukin 2 and soluble interlcukin 2 receptors in endoscopical mucosal biopsy specimens with active inflammatory bowel discase. Gut $1992 ; 33$ : 55-8.

22. Stevens C, Walz G, Lanker B, Singaram C, Limpan $\mathrm{M}$, Strom TB. Interlcukin 6 , interlcukin $1 \beta$ and tumor necrosis factor $\alpha$ expression in inflammatory bowc discase. Gastroenterology 1990; 98 : A 475. 23. Bracgger CP, Nicholls S, Murch SH Stephens S, Macl)onalds ' $\Gamma$ 'I'. Tumor necrosis factor alpha in stool as a marker of intestinal inflammation. Lancet 1992 ; 339 : 89-91 24. Kusugami K, Matsuura T, West GA, Youngman KR, Rachmilewitz O, Fiocchi C. Loss of interlcukin-2-producing intestinal CI $4{ }^{+}$T-cells in inflammatory bowel discasc. Gastroenterology 1991; 101 1594-605.

25. Schreiber S, MacI)ermott RP, Racdler A, Pinnau R, Bertovich MJ, Nash GS. Increased activation of isolated intestina lamina propria mononuclear cells in inflammatory bowel discasc. Gastroenterology 1991 101 : 1020-30

26. Allison MC, Poulter I,W, Ihillon AP, Pounder RE. Immunohistological studies of surface antigen on colonic lymphoid cells in normal and inflamed mucosa. Comparison of follicular and lamina propria lymphocytes. Gastroenterology 1990 ; 99 : 421-30.

27. Macl)ermott RP, Franklin GO, Jenkins KM, et al. Human intestinal mononuclear cells. I. Investigation of antibodydependent, lectin-induced, and spontancosus cell-mediated cytotoxic capabilitics. Gastroenterology $1980 ; 78: 47-56$.

28. Macl)ermott RP, Bragdon MJ, Jenkins KM, Franklin G, Shedlofsky S, Kodner IJ, et al. Human intestinal mononuclear cells. II. Demonstration of a naturally occurring subclass of $T$ cells which respond in the allogencic mixed leukocytc reaction but do not affect ccll-mediated lympholysis. Gastroenterology $1981 ; 80$ : 748-57.

29. Macl)ermott RP, Bragdon MJ, Kodner IJ, Bertovich MJ, et al. Deficient cellmediated cytotoxicity and hyporesponsiveness to interferon and mitogenic lectin activation by inflammatory bowel discase peripheral blood and intestinal mononuclear cells. Gastroenterology $1986 ; 90: 6-11$.

30. Mestecky J, Cummins L, Russel MW. Selective transport of IgA : cellular and molecular aspects. Gastroenterol Clin North Am 1991; $20: 441-72$

31. Strober W, Harriman GR. The regulation of $\operatorname{lgA}$ B-cell differentiation. Gastroenterol Clin North Am 1991; 20: 473-94. anormale des lymphocytes $\mathrm{T}$ induit une surexpression d'antigènes d'activation, tels que CD25 et CD71 [25], tandis que l'expression du CD45RO, un marqueur des cellules $T$ à mémoire, n'est pas augmentée [26]. Il est intéressant de noter que la fonction cytotoxique des cellules mononucléées intestinales est diminuée et complètement latente, jusqu'à ce que celles-ci soient activées par l'interféron, les lectines ou les autres cytokines [27-29].

L'IgA est normalement l'immunoglobuline dominante, la plus importante du tube digestif, et constitue le premier mécanisme de défense de la muqueuse $[30,31]$. Cependant la sécrétion d'IgA par les lymphocytes B de l'intestin est diminuée au cours des MICI [32, 33], et est beaucoup trop faible pour que l'IgA puisse être la principale immunoglobuline impliquée dans ces maladies. Nous avons trouvé, en revanche, une énorme augmentation de la production d'IgG par les cellules mononucléées de la lamina propria de l'intestin [34-36]. Il est intéressant de noter que les sousclasses d'IgG, sécrétées par la lamina propria dans les rectocolites hémorragiques, ne sont pas les mêmes que celles sécrétées au cours de la maladie de Crohn : les sous-classes IgG1 et IgG3 sont exprimées de façon prépondérante au cours de la rectocolite hémorragique et les sous-classes IgG1 et IgG2, dans la maladie de Crohn [35, 36] (figure 2). Par l'utilisation d'anticorps monoclonaux et des techniques immunohistochimiques, d'autres groupes ont trouvé des résultats similaires, c'est-à-dire une forte augmentation d'IgG1 au cours de la rectocolite hémorragique et d'IgG2 au cours de la maladie de Crohn [37]. Dans une étude multicentrique, le dosage des sous-classes d'IgG sériques a montré une augmentation du taux d'IgG totales lors des poussées de rectocolite hémorragique et de maladie de Crohn, avec prédominance de l'IgG1 sérique pour la rectocolite hémorragique et de l'IgG2 sérique pour la maladie de Crohn [38]. Ces modifications sériques reflètent très vraisemblablement des anomalies immunologiques du tissu intestinal au cours des poussées aiguës des MICI [38]. Ces observations sont intéressantes car les sous- classes d'IgG ont des fonctions très différentes : la production d'IgG1 représente la principale réponse aux protéines et aux anticorps dépendant des cellules $T[35,36]$. En revanche, on trouve des anomalies des sousclasses d'IgG2 et d'IgG4 chez les enfants atteints de méningite, de pneumonie ou d'autres processus infectieux récidivants. Les $\operatorname{IgG} 1$ et IgG3 sont de meilleurs activateurs de la voie du complément et de l'opsonisation que les $\operatorname{IgG} 2$, et sont donc particulièrement capables d'induire des lésions du tractus intestinal. Bien que dans l'intestin normal l'IgA soit la principale immunoglobuline protectrice, au cours des maladies inflammatoires cryptogénétiques de l'intestin, l'IgG, avec sa capacité à fixer le complément, peut être le pivot du déclenchement des lésions intestinales et de l'inflammation (figure 2) [1-3, 34, 35].

Parmi les lésions auto-immunes directes et les réactions à médiation cellulaire des MICI, certaines peuvent constituer des cibles thérapeutiques. La mise en évidence de l'activation des lymphocytes et des macrophages, avec ses conséquences sur la production de cytokines pro-inflammatoires et sur l'immunorégulation, ouvre un champ de recherche nouveau et très prometteur. La disponibilité d'inhibiteurs spécifiques des cytokines et d'antagonistes de leurs récepteurs va permettre d'explorer, tant sur des modèles animaux qu'en clinique, le rôle de ces voies dans l'inflammation intestinale. La compréhension du rôle des IgG et des sous-classes d'IgG dans l'induction des lésions intestinales, l'étude de l'effet des inhibiteurs de la sécrétion d'anticorps sur l'inflammation, seront aussi d'un grand intérêt. La régulation de la fonction des cellules $T$ et de la formation des granulomes par les neuropeptides et les cytokines sécrétées par les cellules inflammatoires de la muqueuse ouvre de nouvelles voies de recherche thérapeutique particulièrement ciblées vers la maladie de Crohn [39, 40].

L'une des conséquences de l'hyperactivation du système immunitaire de la muqueuse est le déclenchement de processus inflammatoires non spécifiques (figure 3). C'est dans ce domaine que se situe l'action de la plupart des 
médicaments actuels, les stéroïdes et le 5-ASA (acide 5-amino-salicylique) [1-3]. Du fait de l'afflux rapide et permanent de granulocytes et de macrophages dans les deux maladies, la rectocolite hémorragique et la maladie de Crohn, de très nombreux médiateurs de l'inflammation sont libérés, en particulier des prostaglandines, des leucotriènes, le facteur d'activation des plaquettes (PAF, platelet activating factor) et des radicaux libres. Ils agressent alors de façon sévère et non spécifique les cellules épithéliales et toute cellule du tractus gastro-intestinal [1-3].

Le premier médiateur de l'inflammation étudié fut la prostaglandine E2, produite en très grande quantité dans les MICI [41]. On avait remarqué que les anti-inflammatoires non stéroïdiens (AINS) et les inhibiteurs de la cyclo-oxygénase pouvaient aggraver l'état de certains patients [1-3]. Ce phénomène était resté inexpliqué jusqu'à ce que soient démontrées l'inhibition de la voie de la lipooxygénase par le 5-ASA et, plus important, l'augmentation notable de la production de leucotriène $\mathrm{B} 4$ au cours des MICI [42, 43]. Le leucotriène $\mathrm{B} 4$, par son puissant chimiotactisme, joue un rôle important dans l'inflammation intestinale. Les antiinflammatoires non stéroïdiens, qui inhibent la voie de la cyclooxygénase, pourraient diminuer la synthèse des prostaglandines protectrices et augmenter celle des leucotriènes destructeurs. Notons que l'étude des prostaglandines, dans les modèles animaux de l'inflammation, a

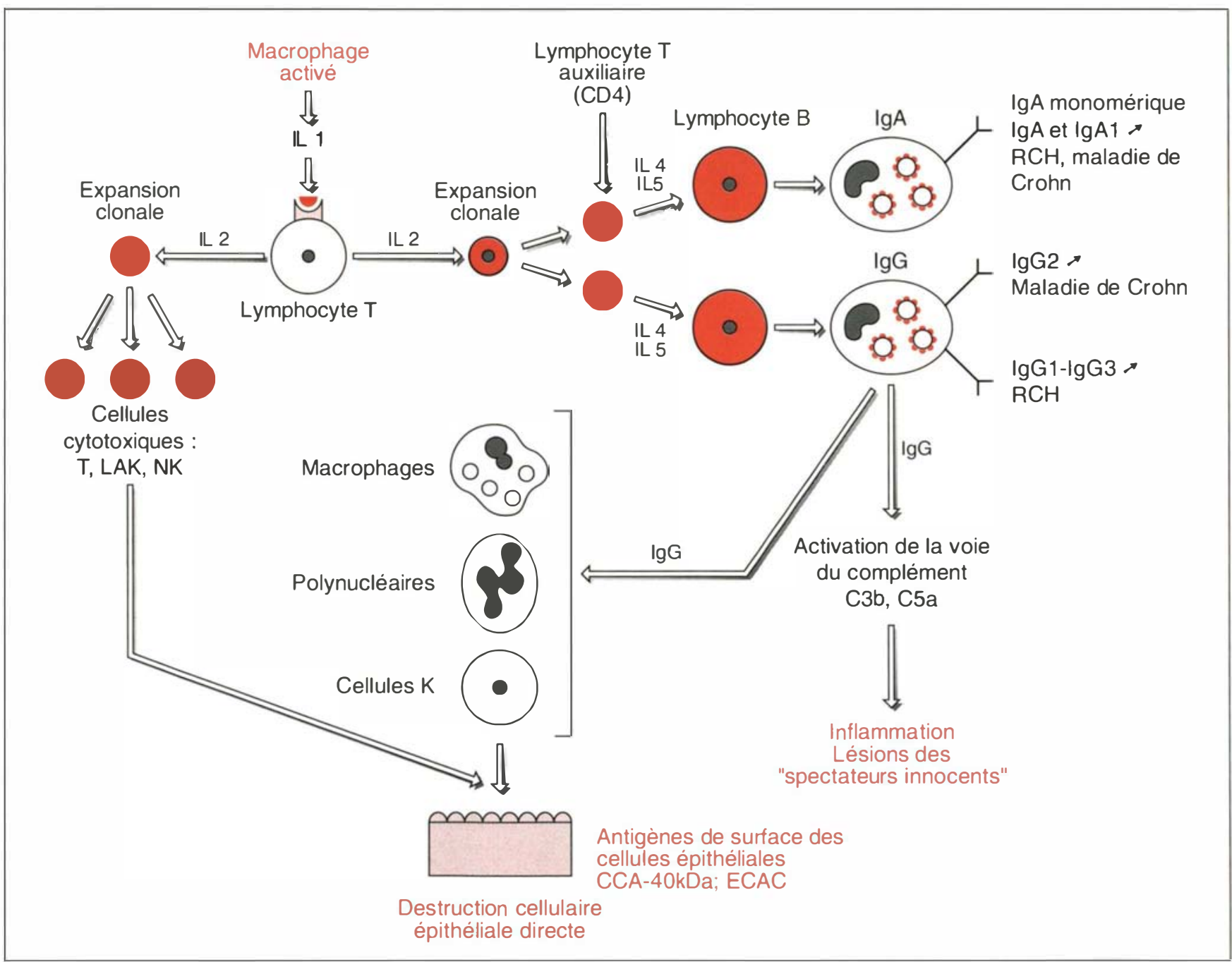

Figure 2. Réactions à médiation cellulaire dans les maladies inflammatoires cryptogénétiques de l'intestin. Après le traitement des antigènes et leur présentation aux lymphocytes $T$ (figure 1) une population de lymphocytes $T$ sensibilisés et activés, capable de sécréter de l'IL2, peut stimuler la prolifération de lymphocytes $T$ auxiliaires spécifiques (CD4). L'accroissement de leur fonction entraîne une sécrétion accrue d'anticorps grâce à la libération de cytokines spécifiques, telles IL4 et IL5. Le développement préférentiel de cellules sécrétrices d'lgA monomérique et d'lgA1 se voit dans la rectocolite hémorragique aussi bien que dans la maladie de Crohn. En revanche, la prolifération importante de plasmocytes exprimant des IgG de sous-classes variées produit des IgG de classes 1 et 3 prédominantes dans la rectocolite hémorragique alors que ce sont les IgG de classes 1 et 2 qui sont prépondérantes dans la maladie de Crohn. (Modifié d'après [47].) 


\section{RÉFÉRENCES}

32. MacI)rmott RP, Beale MG, Alley CI), et al. Synthesis and secretion of $\operatorname{IgA}, \operatorname{Ig} M$, and $\operatorname{Ig} G$ by peripheral blood mononuclear cells, and by human discase states, by isolated human intestinal mononuclear cells, and by human bonc marrow mononuclear cclls from ribs. Ann NY Acad Sci 1983 ; 409 : 498.

33. Macl)ermott RP, Delacroix IL, Nash $\mathrm{GS}$, et al. Evidence for the migration of Bcells secreting monomeric IgA and $\operatorname{Ig} A$ subclass 1 (IgA1) from peripheral compartments into the intestine in inflammatory bowel discase. Gastroenterology 1986 ; 91 : 379-85. 34. Macl)ermott RP, Nash GS, Bertovich $\mathrm{MJ}$, et al. Alterations of $\operatorname{IgM}, \operatorname{IgG}$, and IgA synthesis and secretion by peripheral blood and intestinal mononuclear cells from patients with ulcerative colitis and Crohn's discase. Gastroenterology 1981 ; 81 : 844-52. 35. Scott MG, Nahm MH, Macke K, et al. Spontancous secretion of IgG subclasses by intestinal mononuclear cells : differences between ulcerative colitis, Crohn's discase and controls. Clin Exp Immunol 1986 ; 66 : 209. 36. Macl)ermott RP, Nahm MH. Expression of human immunoglobulin $\mathrm{G}$ subclasses in inflammatory bowel discasc. Gastroenterology 1987 ; 93: 1127-9.

37. Kett K, Rognum TO, Brandtzacg P. Mucosal subclass distribution of IgGproducing cells is different in ulcerative colitis and Crohn's discase. Gastroenterology 1989 ; 93 : 919.

38. Macl)ermott RP, Nash GS, Aucr I(), et al. Alterations in scrum immunoglobulin $G$ subclasses in patients with ulcerative colitis and Crohn's discase. Gastroenterology $1989 ; 96: 764-8$

39. (Ottaway CA. Neuroimmunomodulation in the intestinal mucosa. Gastroenterol Clin North Am 1991; 20 : 511-30.

40. James SP. Mucosal T-cell function. Gastroenterol Clin North Am 1991; 20 : 597-612. 41. Sharon P, Ligumsky M, Rachmilewitz $\mathrm{D}$, et al. Rolc of prostaglandins in ulcerative colitis. Enhanced production during active discase and inhibition by sulfasalazinc. Gastroenterology 1978 ; 75 : 638.

42. Sharon $P$, Stenson WF. Enhanced synthesis of leucotrienc B4 by colonic mucosa in inflammatory bowel discase. Gastroenterology 1984; 86 : 453.

43. Lauritzen K, Laursen ISS, Bukhave K, et al. In vivo effects of orally administered prednisolone on prostaglandin and lcukotrienc production in ulcerative colitis. Gut $1987: 23: 1095$.

44. Fantone JC, Ward PA. Role of oxygenderived free radicals and metabolites in leukocyte-dependent inflammatory reactions. Am J Pathol 1982 ; 107 : 397.

45. Babior BM. The respiratory burst of leukocytes. J Clin Invest 1984 ; 73 : 599-601. 46. Grisham MB, Macl)ermott RP, Deitch EA. Antioxidant enzyme activitics in the human colon. Gastroenterology $1989 ; 96$ : A 185.

47. Macl)ermott RP, Stenson WF. Inflammatory bowel discase. In : Targan SR, Shanahan F, eds. Immunolgy and Immunopathology of the Liver and Gastrointestinal Tract. New

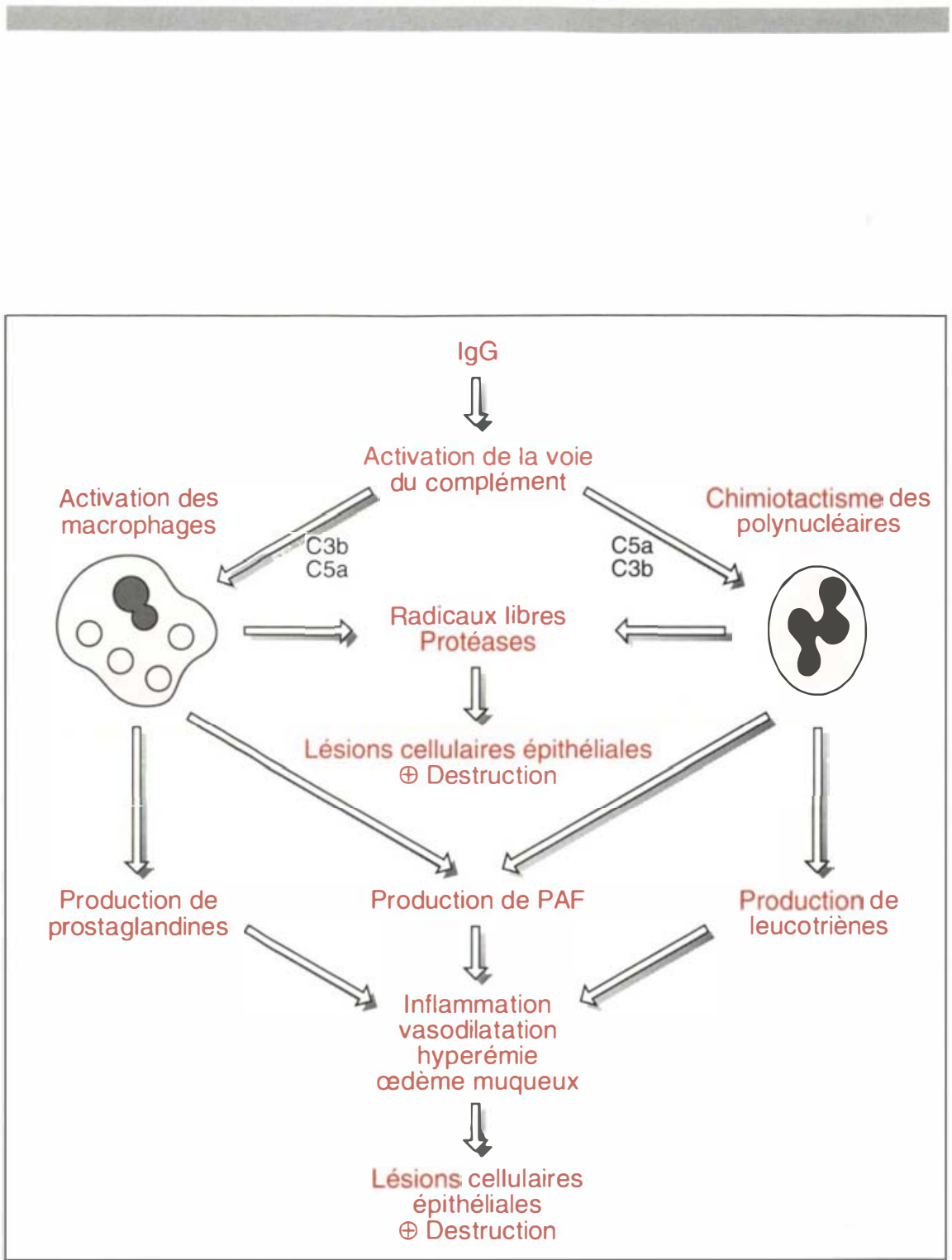

Figure 3. Réactions inflammatoires au cours des maladies inflammatoires cryptogénétiques de l'intestin. Les mécanismes en œuvre dans les $\mathrm{MICl}$ comportent non seulement les réactions spécifiques relayées par les cellules à mémoire (figure 2), mais aussi des réactions inflammatoires non spécifiques qui attaquent l'intestin, spectateur innocent. A la suite de l'augmentation de la sécrétion des IgG dans la rectocolite hémorragique et la maladie de Crohn (figure 2), l'activation du complément peut survenir et libérer des molécules chimiotactiques telles que C3b et C5a qui provoquent l'afflux de polynucléaires et de macrophages. Les polynucléaires à leur tour produisent un excès de leucotriènes dont le chimiotactisme augmente l'afflux de polynucléaires. Les polynucléaires et des macrophages activés libèrent des protéases et $d^{\prime}$ autres enzymes capables d'induire des lésions intestinales. La production et la libération de radicaux libres aggravent à leur tour les lésions des cellules intestinales. Les macrophages activés produisent et relâchent une quantité de médiateurs de l'inflammation, dont des prostaglandines et le facteur d'activation des plaquettes (PAF). Ces médiateurs entraînent une vaso-dilatation, une augmentation de la perméabilité vasculaire, l'hyperhémie et l'œdème de la muqueuse. Ce sont les médiateurs de la réponse inflammatoire qui sont responsables de la majorité des signes pathologiques cliniques des $\mathrm{MICl}$. Le processus inflammatoire pourrait être aussi un aspect de la réaction de défense normale vis-àvis de microorganismes pathogènes, d'agents destructeurs ou d'antigènes déclenchants. (Modifié d'après [47].) 
montré leur effet protecteur sur la muqueuse intestinale. Il est donc probablement important de connaître quelles voies de la cascade métabolique de l'acide arachidonique sont bloquées par les agents pharmacologiques. Des médicaments tels que l'azulfidine, l'olsalazine et autres composés 5-aminosalicylés (mésalazine), qui bloquent préférentiellement la voie de la lipo-oxygénase, semblent augmenter la production des médiateurs de l'inflammation de type protecteur (prostaglandines) et diminuer celle des médiateurs de l'inflammation de type destructeur (leucotriènes). Les modes d'action des stéroïdes sont très divers, l'un d'entre eux pourrait être la diminution de la production d'acide arachidonique endogène à partir des phospholipides membranaires, induisant à la fois une diminution des prostaglandines et des leucotriènes [43]. Il nous faut connaître beaucoup mieux la physiologie des cellules intestinales sécrétant les médiateurs de l'inflammation; l'étude des effets des leucotriènes C4, D4 et E4 sur la perméabilité vasculaire et la contraction du muscle lisse vient tout juste de commencer.

Le 5-ASA piège les radicaux libres, il abaisse aussi leur production. Les radicaux libres sont produits en grande quantité lors des afflux massifs de macrophages et de granulocytes dans les lésions intestinales inflammatoires [44, 45]. Ils sont normalement neutralisés par une série d'enzymes (superoxyde dismutase, catalase, glutathion peroxydase, etc.) et ne provoquent pas de lésion tissulaire. Les taux d'enzymes protecteurs, y compris ceux de superoxyde dismutase et de glutathion peroxydase, sont étrangement bas dans la muqueuse intestinale normale [46]. Le tractus gastro-intestinal représente donc un milicu dans lequel un grand nombre de radicaux libres sont susceptibles de facilement déborder les mécanismes de protection normaux.

Un effort de recherche est nécessaire pour trouver comment inhiber la transition entre les phénomènes inflammatoires aigus, qui constituent un mécanisme normal de protection et le développement de lésions chroniques à médiation cellulaire. Il faut continuer à chercher comment interférer avec cette attraction continue et implacable des macrophages et des granulocytes dans les lésions inflammatoires de l'intestin. De nouvelles voies thérapeutiques pour les maladies intestinales inflammatoires s'ouvriront dès que l'on parviendra à modifier la production des leucotriènes et des prostaglandines, en inhibant la phospholipase A2, en utilisant les huiles de poisson, les inhibiteurs de la voie de la lipo-oxygénase, les inhibiteurs des prostaglandines. Il est important, pour traiter les maladies intestinales inflammatoires, de progresser aussi dans la compréhension de la biologie des radicaux libres pour inhiber leur production et mettre au point de meilleurs pièges. En particulier, l'effet du PAF et de ses antagonistes est à étudier sur modèles animaux et en essais cliniques.

Au total, dans la cascade d'événements qui conduisent aux processus inflammatoires présents dans les maladies intestinales inflammatoires, la production d'immunoglobulines, contrôlée par les cytokines et les cellules, peut être un facteur important car non seulement les immunoglobulines déclenchent l'activation du complément mais elles activent aussi les macrophages (figures 1, 2, et 3). De nombreux facteurs peuvent être responsables du large afflux des macrophages et des granulocytes, dont les cytokines (II 8 et d'autres), l'activation du complément et le leucotriène B4. Une fois réalisé cet afflux cellulaire, l'augmentation manifeste de la production de radicaux libres, de protéases, du leucotriène $\mathrm{B} 4$, du PAF et autres médiateurs (figure 3) conduit aux processus terminaux de l'inflammation que nous voyons s'exprimer cliniquement.

Au cours des dix dernières années, on a fait de grands progrès dans la compréhension des modifications immunologiques observées dans ces maladies. De plus, la mise au jour des mécanismes d'action immunopharmacologi ques des médicaments nous a aidés à développer de nouvelles voies thérapeutiques. A mesure que progresseront nos connaissances en immunologie fondamentale et sur les mécanismes de l'inflammation, de nouvelles et passionnantes stratégies thérapeutiques vont se dessiner pour les malades souffrant de rectocolite hémorragique et de maladie de Crohn

\section{Summary}

Alterations of the mucosal immune system in inflammatory bowel disease

The immunopathology of inflammatory bowel disease consists of a sequence of immunological steps that begin with initial antigen processing events. A major focus has recently been placed on bacterial cell wall products (peptidoglycans, formyl-methionyl-leucyl phenylalanine, lipopolysaccharides). These products nonspecifically induce an intense activation of macrophages, granulocytes, and lymphocytes. Currently, therefore, the working hypothesis is that in inflammatory bowel disease, ubiquitous and common bacterial cell wall products capable of initiating an inflammatory immune response, in a genetically predisposed individual, activate a sequence of immunologic processes that are not appropriately downregulated. Initiating events then lead to macrophage activation, with the resultant production of large amounts of IL1, Il6, Il8, and TNF- $\alpha$. Subsequent cell-mediated immunologic events involve the mucosal immune system, and lead to the chronic intestinal destruction associated with inflammatory bowel disease.

\section{TIRÉS A PART}

R.P. Macl)ermott 